\title{
Relación entre inteligencia emocional y riesgo de adicción a redes sociales en estudiantes de educación superior no universitaria de Lima Metropolitana
}

\author{
Relationship between emotional intelligence and risk of social networking addiction in technical higher \\ education students from Lima
}

Alejandra Jóse Rivera-Véliz ${ }^{1}$, Elizabeth Dany Araujo-Robles ${ }^{1}$

\section{RESUMEN}

El objetivo fue identificar la relación entre la inteligencia emocional y el riesgo de adicción a las redes sociales en un grupo de estudiantes de un instituto de educación superior no universitaria de Lima. El estudio se realizó en 150 estudiantes de entre 16 y 27 años. Se utilizaron el Trait Meta-Mood Scale 24 y el Cuestionario de Adicción a Redes Sociales (ARS). La correlación entre las puntuaciones globales fue baja y no significativa $(r=-, 04 ; p=, 625)$, pero se hallaron correlaciones negativas entre claridad emocional y reparación emocional con indicadores de obsesión por las redes sociales. En cambio, la atención emocional correlacionó positivamente con indicadores de falta de control y uso excesivo de las redes sociales. Estos resultados apuntan a la conclusión de que la claridad emocional y la reparación emocional son componentes fundamentales de la inteligencia emocional, definida como habilidad, en tanto que la atención emocional está relacionada no propiamente con la habilidad de auto-monitoreo emocional, sino más bien con la tendencia a realizar este auto-monitoreo, en particular en individuos con malestar emocional.

PALABRAS CLAVE: inteligencia emocional, adicción a redes sociales, estudiantes.

\section{SUMMARY}

The objective was to identify the relationship between emotional intelligence and risk of social network addiction in a group of students from a technical higher education school from Lima. The sample was constituted by 150 students aged 16 to 27 years. The Trait Meta-Mood Scale 24 and the Questionnaire of Social Networking Addiction were used. The correlation between the full scores was low and not significant $\left(r_{s}=-.04 ; p=.625\right)$, but negative correlations between emotional clarity and emotional repair with indicators of obsessions for the social networking were found, as well as positive correlations between emotional attention with indicators of lack of control and excessive use of social networking. These results lead to the conclusion that emotional clarity and emotional repair are fundamental components of emotional intelligence, as an ability, whereas emotional attention is not mainly related to an ability to self-monitoring one's emotional states, but to a propensity to carry on this self-monitoring, particularly in individuals with emotional discomfort.

KEY WORDS: emotional intelligence, social network addiction, students.

\footnotetext{
Universidad Peruana Cayetano Heredia. Lima, Perú.
} 


\section{INTRODUCCIÓN}

El desarrollo de nuevas tecnologías comunicativas ha permitido que en distintas sociedades las personas resuelvan diversos problemas cotidianos de manera rápida, lo cual es muy notorio en la población joven, que cuenta con una mayor facilidad para su empleo. Entre esas tecnologías destacan las redes sociales, las cuales son consideradas como espacios virtuales que hacen posible la interacción con otras personas (Echeburúa \& Requesens, 2012). Según datos de IPSOS (2018), el 54\% de la población peruana son usuarios de redes sociales; por su parte el Instituto Nacional de Estadística e Informática [INEI] (2018) informó que entre los jóvenes de 17 a 24 años de Lima, la frecuencia diaria en uso de internet, incluyendo el uso de redes sociales, ha aumentado significativamente en los últimos años, en un $48 \%$ desde el 2010 hasta 2018. Este incremento en el uso de internet se puede deber a diversas causas, siendo la principal la conexión constante a las redes sociales, seguida de acceder a información académica o profesional y para realizar actividades de entretenimiento (Fowks, 2017).

La participación en las redes sociales posibilita la apertura a un grupo social amplio y diverso, según lo menciona Cruz (2017), y genera mayores oportunidades para expresar ideas y emociones. Sin embargo, el uso de las redes sociales puede volverse disfuncional. Algunas personas pueden buscar en las redes sociales una compensación frente a la insatisfacción debida a una pobre comunicación familiar y el hecho de no tener amigos (Echeburúa \& Requesens, 2012). El uso excesivo, en algunos casos, puede conducir además a un proceso adictivo. Un empleo de las redes sociales que puede considerarse como adictivo se caracteriza por un uso excesivo, falta de control e indicadores de abstinencia (ansiedad, irritabilidad, depresión) y de tolerancia (aumento de tiempo en el uso de ellas para producir el mismo efecto emocional), así como por afectar de manera negativa en la vida diaria de la persona (por ejemplo, deteriorando la relación con familiares y amigos) y el rendimiento académico o laboral (Andreassen, 2015; Chóliz \& Marco, 2012; Echeburúa \& De Corral, 2010; Kuss \& Griffiths, 2011).

Con base en la definición de adicción a sustancias en el manual DSM-IV y lo referido por Escurra y Salas (2014), se entiende como adicción a las redes sociales un patrón desadaptativo de consumo de redes sociales que conlleva un deterioro o un malestar clínicamente significativo, manifestado por la preocupación constante en los sucesos en las redes sociales, la incapacidad para regular la cantidad de tiempo conectado en redes sociales, el descuido en otras actividades de importancia debido a ello, y el uso excesivo de tiempo en redes sociales. La presencia de estos indicadores puede considerarse como una medida del riesgo de adicción a las redes sociales. En la construcción de un cuestionario para medir tales indicadores, Escurra y Salas (2014) determinaron que los mismos se agrupaban en torno a tres factores:

1. Obsesión: Implica pensamientos constantes en torno a conectarse a las redes sociales y los sentimientos de ansiedad o desasosiego experimentados cuando no se está haciendo uso de éstas.

2. Falta de control personal: Se refiere a la preocupación por el tiempo destinado a estar conectado y por las actividades que se descuidan a causa de las redes sociales.

3. Uso excesivo de las redes sociales: Corresponde a la dificultad para limitar el tiempo de permanencia en estar conectado a las redes sociales.

Los estudios realizados en el Perú en escolares y universitarios revelan en general niveles bajos en estos indicadores. Araujo (2016) tuvo como muestra a 200 estudiantes de diferentes carreras de una universidad de Lima. Como resultado se obtuvo en promedio bajos niveles en las tres dimensiones, pero los varones tuvieron puntajes más altos que las mujeres en el aspecto de obsesión por las redes sociales. Padilla y Ortega (2017), en una muestra de estudiantes de la carrera de psicología de una universidad de Lima obtuvieron niveles bajos y moderados de indicadores de adicción a redes sociales.

En una condición de dependencia, y de modo análogo a lo que sucede con el abuso de sustancias, el uso excesivo de las redes sociales constituye una forma de "liberación emocional" frente al estrés, la ansiedad y la depresión. Las personas con este tipo de dependencia muestran diversos indicadores de malestar psicológico (e. g., Caplan \& High, 2011; Echeburúa \& De Corral, 2010; Griffiths et al., 2014), es decir, un patrón emocional desadaptativo (Charro et al., 2012). Padilla y Ortega (2017) hallaron en una muestra de estudiantes universitarios en Lima una relación significativa entre sintomatología depresiva y las dimensiones de adicción a redes sociales. Otro estudio mostró que la adicción a las redes sociales y a internet está positivamente relacionada con rasgos como el neuroticismo, la ansiedad social y el apego ansioso y negativamente asociada con autoestima 
(Peris et al., 2018). Por otro lado, Delgado et al. (2016) en una muestra de 1405 estudiantes de una universidad pública y una particular de Lima Metropolitana encontraron que quienes cuentan con habilidades sociales, posiblemente no se caractericen por tener una adicción a redes sociales.

Se ha observado que, en algunos casos, el propio medio familiar puede propiciar condiciones que podrían derivar en un uso adictivo de las redes sociales. Por ejemplo, Haro (2018) encontró en estudiantes y egresados universitarios de Lima, que una familia flexible y receptiva a la comunicación virtual, puede tener gran apertura al uso frecuente de las redes sociales, y, por ende, ocasionar en los jóvenes una obsesión por su uso (pensamientos recurrentes).

Por ello, como estrategias de prevención, según Ramón-Cortés (2010), los padres deben enseñar a sus hijos a modular el uso de las redes sociales, deben ayudarlos a que desarrollen habilidades comunicativas y sociales cara a cara, además de limitar el tiempo de uso de la tecnología, fomentar la relación interpersonal con otros, potenciar aficiones como arte o lectura, estimular el deporte y otras actividades recreativas, así como la comunicación entre los miembros de la familia y desarrollar actividades grupales. Es también importante fortalecer el autoconcepto, desarrollar la capacidad de resolución de problemas e informar a los jóvenes sobre las nuevas tecnologías y la peligrosidad de compartir datos personales en ellas (Echeburúa \& De Corral, 2012).

Una situación de dependencia o de riesgo frente a la dependencia de las redes sociales podría comprenderse desde la perspectiva de la teoría de la inteligencia emocional. Cuando un individuo cuenta con un adecuado nivel de inteligencia emocional, se caracteriza por tener habilidades personales, emocionales y sociales que le van a permitir la adaptación y el enfrentamiento exitoso a demandas del contexto, brindándole bienestar general y salud emocional. Una persona que se encuentra permanentemente pendiente de las redes sociales y descuida otros ámbitos significativos de su vida puede tener en baja medida algunas habilidades que forman parte de la inteligencia emocional, como es la capacidad de regulación de estados afectivos negativos (Echeburúa \& De Corral, 2010). Por ende, tal individuo posiblemente cuenta con menos recursos personales y emocionales para afrontar en forma efectiva los problemas cotidianos.
Salovey y Mayer (1990) y Mayer \& Salovey (1992) definieron a la inteligencia emocional como la capacidad de identificar, manejar y regular emociones, tanto propias, como de otros, incluyendo procesos de dirección a nivel emocional, de pensamiento y comportamental, lo que permite una adecuación efectiva a las metas personales y a las demandas ambientales. Asimismo, representa el metaconocimiento de los estados y las destrezas emocionales de la persona a través de diversas actividades, que se manifiestan mediante la atención a las propias emociones y los sentimientos, la claridad y reparación de las emociones. Para Mayer y Salovey, la inteligencia emocional, al menos como ellos la definen, contiene todos los requisitos para considerar que se trata de una verdadera inteligencia (Mayer et al., 1999). No obstante, se trata de una inteligencia que correspondería a un nuevo grupo de inteligencias, a las que Mayer y Salovey denominan "calientes", por su impacto en la vida diaria de las personas y que usualmente han sido ignoradas como objeto de estudio en el campo tradicional de la inteligencia (Mayer et al., 2012).

Dada su naturaleza, el contar con bajos niveles de inteligencia emocional puede conllevar la presencia de problemas psicológicos e interpersonales (e. g., Petrides et al., 2016). Por ejemplo, un estudio de Beranuy et al. (2009) muestra que los componentes de atención, claridad y reparación explican parte de la varianza de indicadores de malestar psicológico (medidos con la SCL-90). Por otro lado, se considera también que un eficiente control emocional, como el que puede ser provisto por adecuados niveles de inteligencia emocional, es un importante factor preventivo frente a problemas conductuales, tales como un patrón adictivo (e. g., Shulman et al., 2016).

En función de lo señalado, se considera que un mayor nivel de indicadores de riesgo de adicción estará asociado a un nivel menor en inteligencia emocional. Sin embargo, no hay una completa consistencia en los resultados de los estudios acerca del papel de la inteligencia emocional en las adicciones tecnológicas. Por ejemplo, Van Deursen et al. (2015) no detectaron relaciones entre la inteligencia emocional y la adicción al smartphone, en cambio Vegue (2016) sí halló una asociación entre inteligencia emocional y "uso problemático" del smartphone. Por otro lado, Che et al. (2017) encontraron que la inteligencia emocional, al menos en parte, está negativamente asociada con la adicción al juego online. En un estudio de Yudes et al. (2019) en adolescentes españoles, se 
hallaron correlaciones negativas entre indicadores de inteligencia emocional (medidos con la escala de Wong y Law) y adicción a internet; estas correlaciones fueron bajas y no significativas en varones pero más altas y significativas en las mujeres. Otros estudios también reportaron correlaciones negativas entre inteligencia emocional y uso problemático de internet (e. g., Maddi et al., 2013).

Sin embargo, el uso de internet involucra muchas distintas actividades, lo que no permite apreciar directamente la relevancia de la inteligencia emocional para el desarrollo o mantenimiento de patrones adictivos frente a las redes sociales. Por tanto, otra serie de trabajos se han ocupado más directamente de esta cuestión. Süral et al. (2019) hallaron en adultos una fuerte correlación negativa entre inteligencia emocional y uso problemático de las redes sociales. Otro estudio, en adolescentes, halló esencialmente el mismo resultado (Kirkaburun et al., 2019). Una característica de los trabajos realizados es la amplia variedad de instrumentos empleados en la medición de la inteligencia emocional y de la adicción o uso problemático de las redes sociales. Pero, en general, existen pocos trabajos acerca de la relación entre inteligencia emocional y adicción a redes sociales, frente a la cantidad de los que se ocupan de la relación e inteligencia emocional y adicción a internet o a otras tecnologías.

En un estudio realizado en la localidad de Tingo María (Perú), Jaramillo (2018) halló una relación no lineal y con tendencia negativa entre inteligencia emocional e indicadores de adicción a las redes sociales, concluyendo que la inteligencia emocional en estudiantes puede ser considerado en cierta medida como un factor predictivo del desarrollo de una adicción a redes sociales. En el estudio de Jaramillo se investigó una muestra de 113 estudiantes de carreras técnicas en una ciudad del interior del país, por lo que se juzgó conveniente investigar la relación entre estas variables en una muestra de mayores dimensiones proveniente de la capital del país. Por otro lado, si bien, por lo general se encuentra una relación inversa entre inteligencia emocional y estados emocionales negativos, existe evidencia de que el factor de atención emocional correlaciona positivamente con algunos de dichos estados negativos, como la ansiedad y la depresión (e. g., Barraza-Lopez et al., 2017; Fernández \& Barraca, 2005). Dado que, a su vez, tales estados negativos con frecuencia están positivamente asociados con indicadores de adicción a las redes sociales, cabe inferir que el factor de atención podría estar también positivamente vinculado con dichos estados. De ser este el caso, ello plantearía cuestiones relativas al rol de la atención emocional como un componente de la inteligencia emocional. El presente estudio se propuso, por tanto, identificar la relación entre inteligencia emocional y riesgo de adicción a redes sociales en un grupo de estudiantes de un instituto técnico de Lima Metropolitana y evaluar tales relaciones a nivel de los tres componentes de la inteligencia emocional.

\section{MÉTODO}

La población estuvo conformada por 213 estudiantes matriculados durante el año 2019 en las carreras de hotelería y turismo y diseño de moda de un instituto de profesiones empresariales del distrito de Santiago de Surco (Lima, Perú) con edades entre 16 y 27 años. Inicialmente se proyectó obtener datos de toda esta población; sin embargo, 12 estudiantes menores de edad no pudieron participar por falta de consentimiento por parte de sus padres o tutores y en otros casos no se pudo obtener los datos por inasistencia a las sesiones de evaluación. La muestra final estuvo conformada por 150 estudiantes. Todos los integrantes de la muestra firmaron el consentimiento o asentimiento informado, según correspondiera.

\section{Instrumentos}

\section{Trait Meta-Mood Scale (TMMS-24).}

Este instrumento fue creado originalmente por Salovey et al. (1995) en Estados Unidos, fue traducido al español por Fernández-Berrocal et al. (2004) y adaptado a la población peruana por Carrasco (2017). Esta escala está conformada por 24 ítems agrupados en tres dimensiones: Atención emocional, Claridad Emocional y Reparación de las Emociones. Se responde mediante una escala tipo Likert de cinco alternativas, desde 1="nada de acuerdo", a $5=$ "totalmente de acuerdo". A mayor puntaje sumado, mayor es el nivel de inteligencia emocional, siendo el puntaje máximo 120.

La adaptación peruana (Carrasco, 2017) conservó el contenido, cantidad y orden de los ítems de la versión española. La validez se evaluó mediante la correlación ítem-test por dimensión, obteniéndose elevadas correlaciones; de este modo en la dimensión Claridad Emocional el coeficiente más bajo fue de .839 , en Atención Emocional el coeficiente más bajo fue de $.799 \mathrm{y}$, por último, en la dimensión de 
Reparación de las Emociones, el coeficiente más bajo fue de .855 . Referente a la confiabilidad, se obtuvo en Atención Emocional un alfa de .837, en Claridad Emocional, .866, y en la dimensión de Reparación de las Emociones, .883.

\section{Cuestionario de Adicción a Redes Sociales (ARS).}

Fue creado y validado en una muestra de universitarios por Escurra y Salas (2014) en Lima, sobre la base de las características de la adicción, según el DSM-IV. Está conformado por 24 ítems que consisten en afirmaciones acerca de los sentimientos y conductas en torno al uso de las redes sociales y que están agrupados en tres dimensiones: Obsesión por las Redes Sociales, Falta de Control Personal y Uso Excesivo de Redes Sociales. Cada ítem se responde mediante una escala graduada tipo Likert con cinco alternativas de respuesta, desde $0=$ "nunca" hasta 4="siempre". Todos los ítems son de calificación directa, excepto el 13. Una mayor puntuación indica una intensidad mayor en las dimensiones respectivas y en el constructo global de adicción. La confiabilidad de las puntuaciones es bastante buena, con alfas correspondientes a las dimensiones entre .88 y .92 . La estructura tridimensional mencionada fue establecida y confirmada mediante análisis factorial.

\section{Procedimiento}

Se realizó la aprobación del estudio por parte del Comité Institucional de Ética, para luego tramitarse los permisos necesarios con las autoridades y los docentes de la institución educativa para ingresar a las aulas y aplicar los instrumentos a los estudiantes. Después de ello, se explicó a los participantes los objetivos del estudio y se les entregó los dos instrumentos. Al culminar la aplicación de pruebas, se brindó a los participantes información acerca del uso riesgoso de las redes sociales y la importancia del desarrollo de habilidades de inteligencia emocional y se les entregó trípticos informativos acerca del tema.

\section{Análisis de datos}

En primer lugar, se calcularon los estadísticos descriptivos de todas las variables. En esos cálculos se incluyó la puntuación media por ítem, que resulta de dividir la puntuación media en un factor entre el número de ítems de dicho factor, con el fin de producir una medida aproximada de la puntuación promedio otorgada por los participantes a los ítems del factor en cuestión. También se evaluó la normalidad o no de las variables mediante el estadístico de KolmogorovSmirnov. Finalmente, para el análisis de correlación, se hizo uso del coeficiente de correlación no paramétrico de Spearman.

\section{RESULTADOS}

En la Tabla 1 se muestra la media y los resultados de la prueba de normalidad de todas las variables. Los resultados de la prueba de Kolmogorov-Smirnov indican que la mayoría de las puntuaciones tienen distribución normal, excepto el caso de la claridad emocional y la obsesión por las redes sociales. La Tabla 1 también indica la puntuación media por ítems. Considerando que la puntuación en cada ítem en inteligencia emocional varía de 1 a 5 , los resultados indican un nivel de puntuación algo por encima del punto medio, que es 3 . En el caso de la adicción a las redes sociales, considerando que la puntuación por ítem varía de 0 a 4, con el 2 como valor medio, puede decirse que predominan las puntuaciones bajas.

Por otro lado, la representación gráfica de las puntuaciones totales indica claramente una distribución normal de las puntuaciones de inteligencia emocional (Figura 1) pero cierta tendencia a las puntuaciones bajas en indicadores de adicción a las redes sociales (Figura 2).

Tabla 1.

Media, Puntuación Media por Ítem y Prueba de Normalidad de las Variables de Estudio

\begin{tabular}{lcccc}
\hline & $\boldsymbol{M}$ & $\boldsymbol{M} / \boldsymbol{I}$ & $\boldsymbol{Z}$ & $\boldsymbol{p}$ \\
\hline $\mathrm{AE}$ & 25.77 & 3.22 & 0.989 & .282 \\
$\mathrm{CE}$ & 26.01 & 3.25 & 3.195 & $<.001$ \\
$\mathrm{RE}$ & 28.56 & 3.57 & 0.954 & .323 \\
$\mathrm{IE}$ & 78.99 & & 0.769 & .595 \\
$\mathrm{OB}$ & 9.17 & 0.92 & 1.524 & .019 \\
$\mathrm{FC}$ & 7.61 & 1.27 & 1.164 & .133 \\
$\mathrm{UE}$ & 12.25 & 1.53 & 0.781 & .576 \\
ARS & 20.08 & \multicolumn{4}{c}{1.010} & .260 \\
\hline Nota. $M / I=$ puntuación media por ítem; $Z=$ estad́stico de \\
Kolmogorov-Smirnov, AE $=$ atención emocional; CE $=$ claridad \\
emocional; RE = reparación emocional; IE $=$ inteligencia emocional \\
total; OB = obsesión por las redes sociales; FC = falta de control; UE \\
$=$ uso excesivo; ARS = adicción a las redes sociales total.
\end{tabular}

Antes de calcular la correlación entre las puntuaciones globales se procedió a establecer el gráfico de dispersión. Tal como se muestra en la Figura 3, las puntuaciones se encuentran muy dispersas previendo que no existe correlación entre las puntuaciones totales de inteligencia emocional y adicción a las redes sociales $\left(r_{s}=-.04 ; p=.625\right)$. 
Figura 1.

Distribución de las Puntuaciones Totales de Inteligencia Emocional

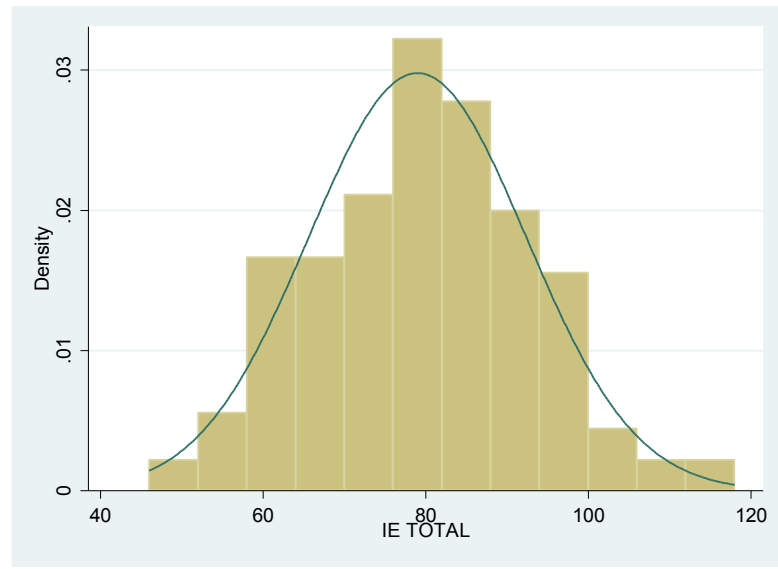

Figura 2.

Distribución de las Puntuaciones en Adicción a las Redes Sociales

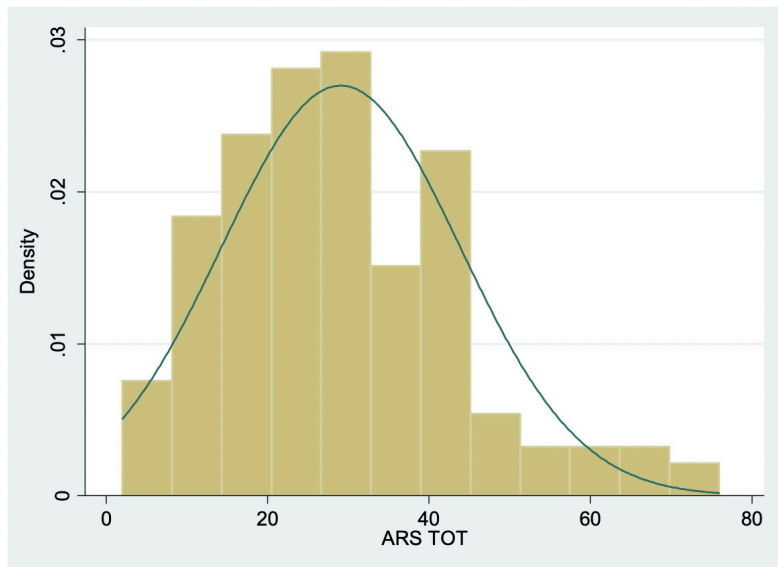

Figura 3.

Gráfico de Dispersión de las Puntuaciones de Inteligencia Emocional y Adicción a Redes Sociales

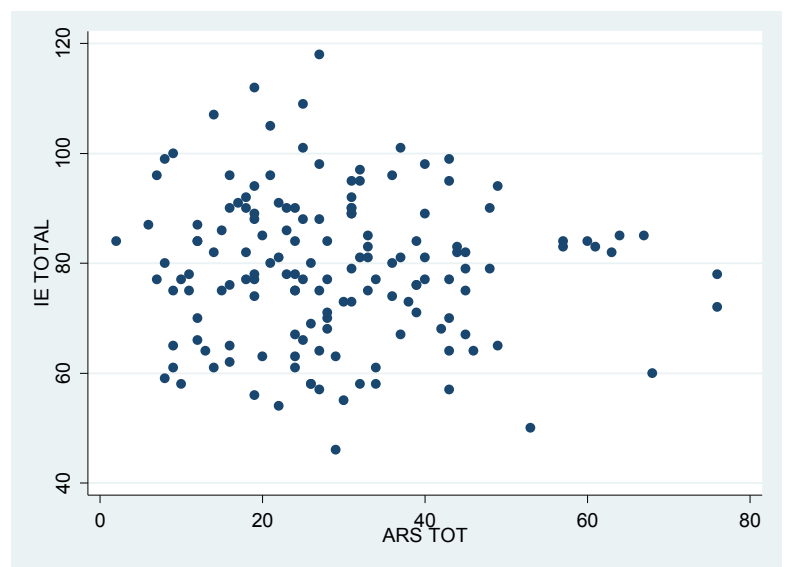

Tabla 2.

Correlaciones Spearman entre las Dimensiones de Inteligencia Emocional y Adicción a las Redes Sociales

\begin{tabular}{cccc}
\hline & $\mathbf{A E}$ & $\mathbf{C E}$ & $\mathbf{R E}$ \\
\hline \multirow{2}{*}{ OB } & $r_{s}=.018$ & $r_{s}=-.237$ & $r_{s}=-.187$ \\
& $p=.187$ & $p=.004$ & $p=.022$ \\
FC & $r_{s}=.211$ & $r_{s}=-.093$ & $r_{s}=-.083$ \\
& $p=.010$ & $p=.258$ & $p=.311$ \\
& $r_{s}=.222$ & $r_{s}=-.143$ & $r_{s}=-.077$ \\
UE & $p=.006$ & $p=.082$ & $p=.349$ \\
\hline
\end{tabular}

Nota. $\mathrm{AE}=$ atención emocional; $\mathrm{CE}=$ claridad emocional; $\mathrm{RE}=$ reparación de las emociones; $\mathrm{OB}=$ obsesión por las redes sociales; $\mathrm{FC}=$ Falta de control; $\mathrm{UE}=$ uso excesivo; $r_{s}=$ Rho de Spearman.

Sin embargo, el examen de las correlaciones a nivel de dimensiones proporciona un cuadro más exacto de la relación entre estas dos variables. La Tabla 2 presenta las correlaciones entre las dimensiones de ambas variables, pudiéndose apreciar que algunas de estas correlaciones son significativas. De esta manera, la dimensión de obsesión por las redes sociales correlaciona negativamente con claridad emocional y con reparación de las emociones, en tanto que la falta de control y el uso excesivo de las redes sociales correlacionan positivamente con atención emocional.

En relación con la dimensión de obsesión por las redes sociales, las correlaciones halladas indican que el pensamiento contantemente enfocado en las redes sociales está vinculado de manera inversa con la capacidad para discriminar y comprender de manera efectiva estados emocionales propios y ajenos y con la capacidad para regular efectiva y positivamente los estados emocionales. Respecto de la dimensión de falta de control, la correlación positiva, aunque baja, con atención emocional indicaría que la preocupación constante que la persona experimente al no tener control del uso de las redes sociales está positiva pero parcialmente asociada con su atención a las emociones y sentimientos de otros. Finalmente, respecto de la dimensión de uso excesivo de las redes sociales, su correlación con atención emocional indica que el tiempo excesivo de uso de las redes sociales y la incapacidad para controlar este uso también se relaciona con la capacidad para identificar y atender emociones propias y de otros de manera efectiva. En general, las correlaciones negativas entre obsesión con las redes sociales y claridad emocional y reparación emocional se encuentran dentro de lo esperado, en tanto que la correlaciones positivas encontradas entre atención emocional y dos de las tres dimensiones de adicción a las redes sociales son menos comprensibles. 
Por otro lado, la falta de control y el uso excesivo no muestran correlaciones significativas con la claridad emocional ni con reparación emocional; es decir, que estos dos conjuntos de indicadores de adicción a las redes sociales no parecen tener vínculo ni con la capacidad para comprender los estados emocionales ni con la habilidad para regularlos.

\section{DISCUSIÓN}

Las puntuaciones en inteligencia emocional tuvieron una distribución normal, lo que, en cierta manera, coincide con hallazgos comunes reportados en la literatura. Así puede apreciarse, por ejemplo, en los resultados de Jaramillo (2018) en estudiantes de Tingo María, de López (2018) en universitarios de Lima Metropolitana y de Vegue (2016) en adultos. En cuanto al nivel encontrado en los indicadores de adicción a redes sociales, las puntuaciones encontradas sugieren un bajo riesgo de adicción, lo que también coincide con resultados anteriormente reportados. Así, por ejemplo, Padilla y Ortega (2017) hallaron un nivel bajo y moderado de indicadores de adicción a redes sociales. Del mismo modo, Araujo (2016) reportó niveles relativamente bajos en las tres dimensiones, en universitarios en el Perú.

La correlación entre las puntuaciones globales de inteligencia emocional e indicadores de adicción a las redes sociales fue bastante baja, aunque sí se hallaron dos correlaciones positivas y dos negativas al realizar un examen al nivel de las dimensiones. Estos resultados corroboran, en general, hallazgos anteriores obtenidos en el Perú respecto de la baja correlación entre estas dos variables (e. g., Jaramillo, 2018). Sin embargo, en otros estudios (Kirkaburun et al., 2019; Süral et al., 2019) sí se aprecian correlaciones significativas entre inteligencia emocional y uso problemático de las redes sociales, tanto en adultos como en adolescentes. Un aspecto común en los estudios de carácter psicosocial tiene que ver con la dificultad de replicar resultados, lo que, a su vez, puede deberse a muchos factores, incluyendo los instrumentales; es decir, la variedad de instrumentos empleados puede reducir la equivalencia entre los distintos estudios que se comparan. El caso de la inteligencia emocional es particularmente ilustrativo de este problema, dada la diversidad de enfoques teóricos y de medición desarrollados en torno a este término (e. g., Peña-Sarrionandia et al., 2015; Webb et al., 2013). De este modo, se considera al menos dos distintos modelos teóricos: el modelo mixto, que incluye habilidades y variables de personalidad (e. g., Bar-On, 2006) y el modelo de habilidad, que sólo incluye aspectos cognitivos (e. g., MacCann, 2014; Mayer et al., 2008). En cuanto a los modelos de medición, se considera también dos opciones, una vinculada a una medida de desempeño y la otra relacionada con una medida de autopercepción (Brackett et al., 2006). Dadas estas diferencias, es inevitable plantear que los diferentes instrumentos diseñados para medir la inteligencia emocional pueden estar evaluando constructos distintos y de ahí, posiblemente, parte de la explicación de los resultados tan dispares respecto de la relación entre inteligencia emocional e indicadores de adicción a las redes sociales.

En el caso particular del presente estudio, debe tenerse en cuenta la característica particular del TMMS-24 de ser un instrumento diseñado para medir la inteligencia emocional percibida, más que la inteligencia emocional misma (Extremera \& Fernández, 2007). Este aspecto puede explicar las correlaciones relativamente bajas encontradas entre la inteligencia emocional y los indicadores de adicción a las redes sociales. También es pertinente considerar que las puntuaciones obtenidas en esta segunda variable han sido también bajas, lo que incide en un posible efecto de piso en la correlación con la inteligencia emocional.

De todos modos, al considerarse los resultados por dimensiones se puede tener una visión más clara de la naturaleza de la relación entre estas variables. Los resultados indicaron que el factor de atención emocional correlaciona sólo con falta de control y con uso excesivo de las redes sociales, mientras que la claridad emocional y la reparación emocional correlacionan sólo con la obsesión por las redes sociales. Además, estas últimas dos correlaciones son negativas, como se esperaba, en tanto que las dos primeras son positivas. Algunos autores han sugerido que las tres dimensiones de la inteligencia emocional guardarían entre sí una relación de secuencialidad (e. g., Palmer et al., 2003), lo que implicaría que no son dimensiones por completo independientes sino que alguna de ellas es antecesora de otra. Este concepto podría ayudar a explicar las correlaciones encontradas.

En efecto, podría sugerirse que las que se considerarían las dos fases finales del proceso de regulación emocional, correspondientes a la claridad o comprensión emocional y la reparación o control emocional estarían más vinculadas, pero de manera negativa, solamente a los pensamientos constantes respecto de las redes sociales. El individuo con mejores niveles de comprensión y regulación de sus 
emociones posee mejores recursos personales para enfrentar los estados de ánimo negativos y de esa manera, se encuentra menos propenso a intentar buscar un alivio emocional en el uso de las redes sociales. Estas dos dimensiones de la inteligencia emocional también muestran asociaciones negativas con la falta de control y el uso excesivo; sin embargo, estas correlaciones son demasiado bajas y no significativas, lo cual es probablemente comprensible ya que la falta de control y el uso excesivo se refieren a situaciones de más impacto para el sujeto. Es decir, son situaciones en que la persona no sólo está pensando en las redes sociales, sino que ya está conectada, por lo que incluso si los procesos regulatorios de los estados emocionales son más intensos, no lo son al nivel requerido para que el individuo pueda lograr el equilibrio emocional necesario para controlar el uso excesivo de las redes sociales.

La explicación anterior plantea que los procesos de comprensión y regulación emocional, tienen más impacto en los indicadores de adicción a las redes sociales (aunque limitados a la obsesión por las redes sociales), debido a que son los más "activos", en comparación con el más pasivo de la atención. Sin embargo, es posible otra explicación, según la cual dicho efecto es simplemente el que se espera por el hecho de ser ambos factores componentes de la inteligencia emocional, mientras que la situación de la atención emocional no radica tanto en ser el componente "pasivo", sino, más bien, posiblemente, en que se trata de un aspecto diferente, que no puede ser por completo englobado dentro del concepto de inteligencia emocional.

Este argumento se basa no sólo en las correlaciones positivas entre la atención y los indicadores de adicción, sino también en las correlaciones positivas entre la atención y otros indicadores de disfuncionalidad. Según lo expuesto en la literatura, aquellos individuos que puntúan alto en los factores de falta de control y uso excesivo probablemente se caracterizan por un estado de ánimo negativo y cabría preguntarse si son precisamente éstos individuos (con estados de ánimo negativos) los más atentos a su estado emocional. Un estudio de Fernández-Berrocal et al. (2006), realizado en adolescentes, mostró que los factores de claridad y reparación correlacionaron fuertemente de manera negativa con ansiedad y con depresión, como es lo esperado; en cambio, el factor de atención correlacionó positivamente, aunque a un nivel no significativo, con dichos estados emocionales. Otro estudio, en cambio, sí mostró una correlación positiva significativa entre atención y ansiedad en estudiantes de bachillerato en España (Fernández \& Barraca, 2005). En una revisión, Extremera y Fernández (2005) hallaron que una mejor adaptación psicológica se relaciona con puntuaciones bajas o moderadas en atención emocional. Aun un estudio más reciente, de Barraza-López et al. (2017), en estudiantes de medicina, reveló que la claridad y la reparación se asocian negativamente con síntomas depresivos, mientras que la atención está asociada positivamente con dichos síntomas. Barraza-López et al. también destacan un hecho importante: la dimensión de atención puede estar relacionada con las conductas de rumiación presente en los estados depresivos. Extremera y Fernández (2005) plantearon que esta asociación es más probable cuando el individuo presenta también puntuaciones bajas en claridad y en reparación.

Se podría sugerir, a partir de aquí, que la correlación positiva entre atención emocional y disfuncionalidad emocional indica que la atención emocional no tiene que ver principalmente con un factor de habilidad respecto de las emociones; en este caso, la habilidad para monitorear las emociones propias, sino más bien con una tendencia del individuo a realizar constantemente este auto-monitoreo. En función de este análisis, se podría plantear que la correlación positiva entre atención emocional y descontrol en el uso de las redes sociales indica fundamentalmente que este descontrol está asociado con una mayor tendencia al auto-monitoreo de las emociones. Pero este monitoreo, a su vez, está asociado con síntomas de disfuncionalidad emocional. Es decir, que la explicación de la relación positiva entre atención emocional e indicadores de adicción a las redes sociales radica en que el factor de atención emocional comprende principalmente no una habilidad coherente con el concepto de inteligencia emocional, sino, más bien, una tendencia hacia el auto-monitoreo de los estados emocionales, que es característica de los estados emocionales disfuncionales que, como ya es conocido, están asociados al descontrol en el uso de las redes sociales. En suma, se puede concluir, en primer lugar, que la atención emocional no constituye propiamente una capacidad para detectar estados emocionales, sino solamente la propensión a fijarse en ellos. Esto puede además corroborarse si realizamos una revisión de los ítems que componen esta escala (por ejemplo, en Angulo \& Albarracín, 2018; Espinoza-Venegas et al., 2015; Ocaña et al., 2019), la cual indica que la mayoría de ellos se enfoca en pensar en los sentimientos y la propensión a dejar que los sentimientos influyan en los pensamientos. 
En segundo lugar, se podría sugerir también que mientras la claridad y la reparación pueden ser factores protectores frente a la posibilidad de desarrollar estados emocionales negativos, el grado de atención constituye, más bien, una consecuencia de la intensidad de dichos estados emocionales negativos. En otras palabras, que la correlación positiva entre atención y descontrol en el uso de las redes sociales se puede explicar como una consecuencia de que ambos factores son influenciados a la vez por el estado emocional del individuo. Es más, esta relación es quizá aún más compleja, del tipo U. Sólo a nivel de puntuaciones bajas en atención emocional, un aumento en este factor refleja menor disfuncionalidad, lo que resultaría coherente con la idea de la atención con un componente de la inteligencia emocional. Sin embargo, traspuesto un determinado punto, todavía a nivel de puntuaciones bajas (para preservar las correlaciones positivas observadas), los incrementos en la atención reflejan más bien mayor disfuncionalidad.

Futuros estudios en una población más amplia, con muestras de mayores dimensiones y, sobre todo, mediante un examen más detenido del grado de convergencia del constructo de atención emocional y otras variables podrían proporcionar datos que permitan evaluar las hipótesis que se acaba de plantear sobre la naturaleza de la atención emocional. Por otro lado, el tópico general de la relación entre inteligencia emocional y riesgo de adicción a las redes sociales también podría aclararse mediante el empleo de otras medidas de ambas variables, considerando también las visiones mixta y estrictamente cognitiva de la inteligencia emocional.

\section{Autor de correspondencia:}

Elizabeth D. Araujo-Robles, elizabeth.araujo.r@upch.pe

\section{REFERENCIAS}

Andreassen, C. S. (2015). Online social network site addiction; A comprehensive review. Current Addiction Reports, 2(2), 175-184. http://dx.doi.org/10.1007/ s40429-015-0056-9

Angulo, R., \& Albarracín, A. P. (2018). Validez y confiabilidad de la escala rasgo de metaconocimiento emocional (TMMS-24) en profesores universitarios. Revista Lebret, 10,61-72.https://dx.doi.org/10.15332/ rl.v0i10.2197

Araujo, E. (2016). Indicadores de adicción a las redes sociales en universitarios de Lima. Revista Digital de Investigación en Docencia Universitaria, 10(2), 4858. http://dx.doi.org/10.19083/ridu.10.494
Bar-On, R. (2006). The Bar-On model of emotional-s ocial intelligence (ESI). Psicothema, 18, 13-25.

Barraza-López, R. J., Muñoz-Navarro, N. A, \& BehrensPérez, C. C. (2017). Relación entre inteligencia emocional y depresión-ansiedad y estrés en estudiantes de medicina de primer año. Revista

Chilena de Neuro-Psiquiatría, 55(1), 18-25. http:// dx.doi.org/10.4067/S0717-92272017000100003

Beranuy, M., Oberst, U., Carbonell, \& Chamarro, A. (2009). Problematic internet and mobile phone use and clinical symptoms in college students: the role of emotional intelligence. Computers in Human

Behavior, 25, 1182-1187. http://dx.doi.org/10.1016/j. chb.2009.03.001

Brackett, M. A., Rivers, S. E., Shiffman, S., Lerner, N., \& Salovey, P. (2006). Relating emotional abilities to social functioning: A comparison of self-report and performance measures of emotional intelligence. Journal of Personality and Social Psychology, 91, 780-795. http://dx.doi.org/10.1037/0022-3514.91.4. 780

Caplan, S. E., \& High, A. C. (2011). Online social interaction, psychosocial well-being, and problematic internet use. En K. S. Young \& C. de Abreu (Eds.), Internet addiction. A handbook and guide to evaluation and treatment (pp. 35-53). Hoboken, NJ: John Wiley \& Sons Inc.

Carrasco,J.(2017).RelaciónentreInteligenciaEmocional, Autoeficacia General y Variables Sociodemográficas en pacientes ambulatorios del servicio de psicología de una clínica de Lima Metropolitana. [Tesis de licenciatura, Universidad Peruana Cayetano Heredia]. Repositorio Institucional de la Universidad Peruana Cayetano Herediahttp://repositorio. upch.edu.pe/bitstream/handle/upch/984/Relacion CarrascoTacuri_Jennifer.pdf? sequence= $1 \&$ isAllowed $=\mathrm{y}$.

Charro, B., Meneses, C., \& Del Cerro, P. (2012). Motivos para el consumo de drogas legales y su relación con la salud en los adolescentes madrileños. Revista Española de Drogodependencias, 37(3), 257-268.

Che, D., Hu, J., Zhen, S., Yu, C., Li, B., Chang, X., \& Zhang. W. (2017). Dimensions of emotional intelligence and online gaming addiction in adolescence: the indirect effects of two facets of perceived stress. Frontiers in Psychology, 8, 1206. http://dx.doi.org/ 10.3389/fpsyg.2017.01206

Chóliz, M., \& Marco, C. (2012). Adicción a internet $y$ redes sociales. Tratamiento psicológico. Alianza Editorial

Cruz, L. (2017). Adolescentes y redes sociales en la era digital. [Tesis de licenciatura, Universidad Peruana Cayetano Heredia]. Repositorio Institucional de la Universitat de les Illes Balears. http://dspace. uib.es/xmlui/bitstream/handle/11201/3808/Cruz Diana_Laura.pdf?sequence $=1$. 
Delgado, A., Escurra, L., Atalaya, M., Pequeña, J., Cuzcano, A., Rodríguez, R. E., \& Álvarez, D. (2016). Las habilidades sociales y el uso de redes sociales virtuales en estudiantes universitarios de Lima Metropolitana. Persona, 19, 55-75.

Echeburúa, E., \& De Corral, P. (2010). Adicción a las nuevas tecnologías y a las redes sociales en jóvenes: un nuevo reto. Adicciones, 22(2), 91-96.

Echeburúa, E., \& Requesens, A. (2012). Adicción a las redes sociales y a las nuevas tecnologías en niños $y$ adolescentes. Guía para educadores. Ediciones Pirámide.

Escurra, M., \& Salas, E. (2014). Construcción y validación del Cuestionario de Adicción a Redes Sociales (ARS). Liberabit, 20(1), 73-91.

Espinoza-Venegas, M., Sanhueza-Alvarado, O., RamírezElizondo, N. \& Sáez-Carrillo, K. (2015). Validación de constructo y confiabilidad de la escala de inteligencia emocional en estudiantes de enfermería. Revista Latino-Americana de Enfermagem, 23(1), 139-147. https://dx.doi.org/10.1590/01041169.3498.2535

Extremera, N. \& Fernández, P. (2004). Inteligencia emocional, calidad de las relaciones interpersonales y empatía en estudiantes universitarios. Clínica $y$ Salud, 15(2), 117-137.

Extremera, N. \& Fernández, P. (2005). Inteligencia emocional percibida $\mathrm{y}$ diferencias individuales en el meta-conocimiento de los estados emocionales: Una revisión de los estudios con el TMMS. Ansiedad y Estrés, 11(2-3), 101-122.

Extremera, N. \& Fernández, P. (2007). Una guía práctica de los instrumentos actuales de evaluación de la inteligencia emocional. En: J. M. Mestre \& P.

Fernández (Eds.), Manual de inteligencia emocional (pp. 99-122). Editorial Pirámide.

Fernández, A. \& Barraca, J. (2005). Inteligencia emocional, empatía y competencia social, una investigación empírica con estudiantes de bachillerato. http://jorgebarraca.com/wp-content/ uploads/Inteligencia-Emociona-EmpatiaCompetencia-Social-Una\%20investigacionempirica-con-estudiantes-de-Bachillerato.pdf.

Fernández-Berrocal, P., Alcaide, R. \& Pizarro, D. (2006). The role of emotional intelligence in anxiety and depression among adolescents. Individual Differences Research, 4(1), 16-27.

Fernandez-Berrocal, P., Extremera, N. \& Ramos, N. (2004). Validity and reliability of the Spanish modified version of the Trait Meta-Mood Scale. Psychological Reports, 94, 751-755. http://dx.doi.org/10.2466/ pr0.94.3.751-755

Fowks, C. (2017). Internet en el Perú. DATUM. https:// www.datum.com.pe/new_web_files/files/pdf/ Internet.pdf
Griffiths, M. D., Kuss, D. J., \& Demetrovics, Z. (2014). Social networking addiction: An overview and preliminary findings. En: K. P. Rosenberg \& L. C. Feder (Eds.), Behavioral addictions: Criteria, evidence, and treatment (pp. 119-141). ElsevierAcademic Press.

Haro, E. (2018). Adicción a Redes Sociales y Funcionamiento Familiar en estudiantes universitarios y egresados de Lima Metropolitana. [Tesis de licenciatura, Universidad San Ignacio de Loyola]. Repositorio Institucional de la Universidad San Ignacio de Loyola. http://repositorio.usil.edu.pe/ bitstream/USIL/3684/3/2018_Haro-Arrarte.pdf.pdf

Instituto Nacional de Estadística e Informática (2018). Población de 6 y más años de edad que hace uso del servicio de internet por grupos de edad, frecuencia de uso y según ámbitos geográficos. INEI. https://www. inei.gob.pe/estadisticas/indice-tematico/populationaccess-to-internet/IPSOS (2018). Perfil del usuario de redes sociales. IPSOS. Recuperado de: https:// www.ipsos.com/es-pe/perfil-del-usuario-de-redessociales-2

Jaramillo, M. (2018). Relación entre Inteligencia Emocional con Indicadores de Adicción a las Redes Sociales en estudiantes de un instituto público de Tingo María 2017. [Tesis de Maestria, Universidad Peruana Cayetano Heredia]. Repositorio Institucional de la Universidad Peruana Cayetano Heredia http://repositorio.upch.edu.pe/bitstream/ handle/upch/6685/Relacion JaramilloSanta\%20 Cruz_Maritha.pdf?sequence $=1 \&$ isAllowed $=\mathrm{y}$

Kircaburun, K., Griffiths, M. D. \& Billieux, J. (2019). Trait emotional intelligence and problematic online behaviors among adolescents: The mediating role of mindfulness, rumination, and depression. Personality and Individual Differences, 139, 208-213. http:// dx.doi.org/10.1016/j.paid.2018.11.024

Kuss, D. J., \& Griffiths, M. D. (2011). Online social networking and addiction-A review of the psychological literature. International Journal of Environment Research and Public Health, 8(9), 3528-3552. http://dex.doi.org/10.3390/ijerph8093 528

López, O. (2008). La Inteligencia emocional y las estrategias de aprendizaje como predictores del rendimiento académico en estudiantes universitarios. [Tesis de Maestria, Universidad Nacional Mayor de San Marcos]. Repositorio Institucional de la Universidad Nacional Mayor de San Marcos. http:// cybertesis.unmsm.edu.pe/bitstream/cybertesis/615/1/ Lopez_mo.pdf

MacCann, C., Joseph, D. L., Newman, D. A., \& Roberts, R. D. (2014). Emotional intelligence is a secondstratum factor of intelligence: Evidence from hierarchical and bifactor models. Emotion, 14, 358374. http://dx.doi.org/10.1037/a0034755 
Maddi, S. R., Erwin, L. M., Carmody, C. L., Villarreal, B. J., White, M. \& Gundersen, K. K. (2013). Relationship of hardiness, grit, and emotional intelligence to internet addiction, excessive consumer spending, and gambling. The Journal of Positive Psychology, 8(2), 128-134. http://dx.doi.org/10.1080/17439760.2012.7 58306

Mayer, J. D., Caruso, D. R., Panter, A. T. \& Salovey, P. (2012). The growing significance of hot intelligences. American Psychologist, 67(6), 502-503. https:// dx.doi.org10.1037/a0029456

Mayer, J. D., Caruso, D. R. \& Salovey, P. (1999). Emotional intelligence meets traditional standards for an intelligence. Intelligence, 27(4), 267-298. https://dx.doi.org/10.1016/s0160-2896(99)00016-1

Mayer, J. D. \& Salovey, P. (1993). The intelligence of emotional intelligence. Intelligence, 17, 433-442.

Mayer, J. D., Salovey, P., \& Caruso, D. R. (2008). Emotional intelligence: New ability or eclectic traits? American Psychologist, 63(6), 503-517. http://dex. doi.org/10.1037/0003-066X.63.6.503

Ocaña, J., García, G. A. \& Cruz, O. (2019). Propiedades psicométricas del Trait Meta-Mood Scale TMMS-24 en adolescentes de Chiapas, México. European Scientific Journal, 15(16), 280-294. https://dx.doi. org/10.19044/esj.2019.v15n16p280

Padilla, C. \& Ortega, J. (2017). Adicción a las redes sociales y sintomatología depresiva en universitarios. CASUS, 2(1), 47-53.

Palmer, B., Gignac, G., Bates, T. \& Stough, C. (2003). Examining the structure of the Trait MetaMood Scale. Australian Journal of Psychology, 55, 154-159.

Peña-Sarrionandia, A., Mikolajczak, M., \& Gross, J. J. (2015). Integrating emotion regulation and emotional intelligence traditions: a meta-analysis. Frontiers in Psychology. https://dx.doi.org/10.3389/ fpsyg.2015.00160

Peris, M., Maganto, C. \& Garaigordobil, M.(2018). Escala de riesgo de adicción-adolescente a las redes sociales e internet: fiabilidad y validez (ERA-RSI). Revista de Psicología Clínica con Niños y Adolescentes, 5(2), 3036. https://dx.doi.org/10.21134/rpcna.2018.05.2.4

Petrides, K. V., Mikolajczak, M., Mavroveli, S., Sanchez-Ruiz, M. J., Furnham, A. \& Pérez-González, J. C. (2016). Developments in trait emotional intelligence research. Emotion Review, 8, 335-341. https://dx.doi.org/10.1177/1754073916650493
Ramón-Cortés, F. (2010, enero 3). ¿Internet amenaza el contacto real? El Pais. https://elpais.com/ diario/2010/01/03/eps/1262503611_850215.html

Salovey, P., \& Mayer, J. D. (1990). Emotional intelligence. Imagination, Cognition and Personality, 9, 185-211. https://dx.doi.org/10.2190/DUGG-P24E-52WK6CDG

Salovey, P., Mayer, J., Goldman, S., Turvey, C., \& Palfai, T. (1995). Emotional attention, clarity, and repair: exploring emotional intelligence using the

Trait MetaMood Scale. En: J. D. Pennebaker (Ed.), Emotion, disclosure, and health (pp. 125-154). American Psychological Association.

Shulman, E. P., Smith, A. R., Silva, K., Icenogle, G., Duell, N., Chein, J. \& Steinberg, L. (2016). The dual systems model: Review, reappraisal, and reaffirmation. Developmental Cognitive Neuroscience, 17, 103-117. https://dx.doi.org/10.1016/j.dcn.2015.12.010

Süral, I., Griffiths, M. D., Kircaburun, K. \& Emirtekin, E. (2019). Trait emotional intelligence and problematic social media use among adults: The mediating role of social media use motives. International Journal of Mental Health and Addiction, 17, 336-345. https://dx.doi.org/10.1007/s11469-018002-6

Van Deursen, A. J. A. M., Bolle, C. L., Hegner, S. M., \& Kommers, P. A. M. (2015). Modeling habitual and addictive smartphone behavior. The role of smartphone usage types, emotional intelligence, social stress, self-regulation, age, and gender. Computers in Human Behavior, 45, 411-420. https:// dx.doi.org/10.1016/j.chb.2014.12.039

Vegue, F. (2016). Inteligencia emocional y uso problemático del smartphone en adultos. Pontificia Universidad Comillas. https://repositorio.comillas. edu/xmlui/bitstream/handle/11531/10105/ TFM000412.pdf? sequence $=1$ \&isAllowed $=y$

Webb, C. A., Schwab, Z. J., Weber, M., DelDonno, S., Kipman, M., Weiner, M. R. \& Killgore, W. D. S. (2013). Convergent and divergent validity of integrative versus mixed model measures of emotional intelligence. Intelligence, 41, 149-156. https://dx.doi. org/10.1016/j.intell.2013.01.004

Yudes, C., Rey, L. \& Extremera, N. (2019). Ciberagresión, adicción a internet $\mathrm{e}$ inteligencia emocional en adolescentes: un análisis de diferencias de género. Voces de la Educación, 2, 27-44. https://hal.archivesouvertes.fr/hal-02511693/document 